\title{
Targeting the Wnt/ $\beta$-catenin pathway in primary ovarian cancer with the porcupine inhibitor WNT974
}

\author{
Jonathan D Boone1, Rebecca C Arend', Bobbi E Johnston², Sara J Cooper², Scott A Gilchrist ${ }^{3}$, Denise K Oelschlager ${ }^{4}$, \\ William E Grizzle ${ }^{4}$, Gerald McGwin Jr ${ }^{5}$, Abhishek Gangrade ${ }^{6}$, J Michael Straughn Jr and Donald J Buchsbaum ${ }^{6}$
}

Preclinical studies in ovarian cancer have demonstrated upregulation of the $\mathrm{Wnt} / \beta$-catenin pathway promoting tumor proliferation and chemoresistance. Our objective was to evaluate the effect of the $\mathrm{Wnt} / \beta$-catenin pathway inhibitor, WNT974, in primary ovarian cancer ascites cells. Ascites cells from patients with papillary serous ovarian cancer were isolated and treated with $1 \mu \mathrm{M}$ WNT974 $\pm 100 \mu \mathrm{M}$ carboplatin. Viability was evaluated with the ATPlite assay. The IC 50 was calculated using a dose-response analysis. Immunohistochemistry $(\mathrm{IHC})$ was performed on ascites cells and tumor. Expression of R-spondin 2 (RSPO2), RSPO3, PORCN, WLS, AXIN2, and three previously characterized RSPO fusion transcripts were assessed using Taqman assays. Sixty ascites samples were analyzed for response to WNT974. The ascites samples that showed a decrease in ATP concentration after treatment demonstrated no difference from the untreated cells in percent viability with trypan blue staining. Flow cytometry demonstrated fewer cells in the G2 phase and more in the G1 and S phases after treatment with WNT974. Combination therapy with WNT974 and carboplatin resulted in a higher percentage of samples that showed $\geq 30 \%$ reduction in ATP concentration than either single drug treatment. IHC analysis of Wnt pathway proteins suggests cell cycle arrest rather than cytotoxicity after WNT974 treatment. QPCR indicated that RSPO fusions are not prevalent in ovarian cancer tissues or ascites. However, higher PORCN expression correlated to sensitivity to WNT974 $(P=0.0073)$. In conclusion, WNT974 produces cytostatic effects in patient ascites cells with primary ovarian cancer through inhibition of the Wnt/ $\beta$-catenin pathway. The combination of WNT974 and carboplatin induces cytotoxicity plus cell cycle arrest in a higher percentage of ascites samples than with single drug treatment. RSPO fusions do not contribute to WNT974 sensitivity; however, higher PORCN expression indicates increased WNT974 sensitivity. Laboratory Investigation (2016) 96, 249-259; doi:10.1038/labinvest.2015.150; published online 14 December 2015

More women in the United States die from ovarian cancer than any other gynecologic malignancy. It is estimated that there will be 21290 new cases of ovarian cancer, and 14180 women will die of the disease in $2015 .{ }^{1}$ Over the last 20 years, advances using platinum/taxane doublets, dose-dense chemotherapy, intraperitoneal chemotherapy, and targeted agents have improved response rates of women with ovarian cancer. $^{2-7}$ Despite these advances, no significant improvement in overall survival has been demonstrated. ${ }^{1}$ Patients with advanced disease treated with a platinum/taxane-based chemotherapy will experience partial response rates over $80 \%$ and complete response rates as high as $60 \%{ }^{8}$ Unfortunately, recurrence occurs in about $70 \%$ of patients who have a complete clinical response, and $90 \%$ of patients who recur will develop resistance to platinum-based chemotherapies. ${ }^{5,9}$

More than 33\% of women diagnosed with ovarian cancer have ascites present on initial presentation. ${ }^{10}$ Presence of ascites is associated with a poorer prognosis. The ascites cells are convenient for clinical research but also valuable scientifically because they play a role in metastasis at the time of primary occurrence and disease recurrence and in chemoresistance. ${ }^{10}$

Because of the high recurrence rates and resistance to salvage chemotherapy, researchers have investigated novel targeted therapies to overcome platinum resistance. The Wnt/ $\beta$-catenin pathway is a signaling pathway that mediates ovarian cancer initiation and progression through genes that

\footnotetext{
${ }^{1}$ Department of Obstetrics and Gynecology, Division of Gynecologic Oncology, University of Alabama at Birmingham, Birmingham, AL, USA; ${ }^{2}$ HudsonAlpha Institute for Biotechnology, Huntsville, AL, USA; ${ }^{3}$ University of Alabama at Birmingham School of Medicine, Birmingham, AL, USA; ${ }^{4}$ Department of Pathology, University of Alabama at Birmingham, Birmingham, AL, USA; ${ }^{5}$ Department of Epidemiology, University of Alabama at Birmingham, Birmingham, AL, USA and ${ }^{6}$ Department of Radiation Oncology, University of Alabama at Birmingham, Birmingham, AL, USA

Correspondence: Dr JD Boone, MD, Department of Obstetrics and Gynecology, Division of Gynecologic Oncology, University of Alabama at Birmingham, 17006 th Avenue South, Room 10250, Birmingham, AL 35233, USA.

E-mail: jboone@uabmc.edu

Received 29 July 2015; revised 30 October 2015; accepted 9 November 2015
} 
regulate cell proliferation and apoptosis. ${ }^{11-13}$ Preclinical studies have shown an upregulation of Wnt ligands in ovarian cancer ascites, which suggests an increase in $\mathrm{Wnt} / \beta$ catenin pathway activity. ${ }^{9}$ The $\mathrm{Wnt} / \beta$-catenin pathway has also been shown to contribute to chemoresistance in ovarian cancer as it promotes epithelial-to-mesenchymal transition. ${ }^{14}$

Porcupine (PORCN) is an enzyme that is crucial to Wnt ligand transport, secretion, and activity. ${ }^{15}$ WNT974 (Novartis, Cambridge, MA, USA) is a selective, orally bioavailable PORCN inhibitor that decreases the secretion of Wnt ligands outside the cell leading to a decrease in Wnt ligand cell surface receptor phosphorylation and a decrease in the expression of Wnt target genes. ${ }^{16}$ WNT974 has been shown to suppress growth of Wnt-driven tumors, such as pancreatic and head and neck squamous cell cancer with mutations in Wnt pathway modulators such as Ring finger 43 (RNF43). ${ }^{17}$ The R-spondin (RSPO) protein family is comprised of four secreted growth factors. Secreted RSPO proteins activate Wnt/ $\beta$-catenin signaling, and RSPO mutations can further upregulate $\mathrm{Wnt} / \beta$-catenin signaling. ${ }^{18}$ Cardona et al ${ }^{19}$ found the prevalence of RSPO fusion gene mutations, primarily with RSPO2 and RSPO3, to be as high as $45 \%$ in patients with ovarian cancer. Our primary objective was to evaluate the effect of the Wnt/ $\beta$-catenin pathway inhibitor, WNT974, in primary ovarian cancer ascites cells and evaluate the importance of R-spondins in sensitivity to WNT974.

\section{MATERIALS AND METHODS}

\section{Specimen Collection and Processing}

With IRB approval, ascites was collected from 60 ovarian cancer patients. Forty of those patients also had matched tumor specimens collected. Cells were isolated from ascites samples as previously described. ${ }^{9}$ Cells were stored in $10 \%$ dimethyl sulfoxide (DMSO) and kept frozen in liquid nitrogen for future analysis. A pathologist confirmed that all samples were papillary serous ovarian or primary peritoneal cancer. The viability of the ascites cells was confirmed with trypan blue staining showing $\geq 74 \%$ viability after the cells had been frozen and thawed twice. All experiments were done using cells that had been thawed only once or twice.

\section{Drugs and Antibodies}

WNT974 was obtained from Novartis under a material transfer agreement. A stock solution of $10 \mathrm{mM} \mathrm{WNT974} \mathrm{was}$ diluted in DMSO at a $5 \mathrm{mM}$ concentration and stored at $4{ }^{\circ} \mathrm{C}$. A $25 \mathrm{mM}$ stock solution of carboplatin was prepared in sterile $\mathrm{H}_{2} \mathrm{O}$.

\section{ATPlite Luminescence-Based Assay}

Patient-derived ascites cells were plated in a 96-well ultra-low attachment plate (Corning Costar, Corning, NY, USA) at 2000 cells/well in $45 \mu \mathrm{l} \mathrm{X}$-vivo serum free media containing $20 \mathrm{ng} / \mathrm{ml}$ epidermal growth factor and $10 \mathrm{mg} / \mathrm{ml}$ bovine insulin. They were then treated with $2.5 \mu \mathrm{l}$ of $1 \mu \mathrm{M}$ WNT974, $2.5 \mu \mathrm{l}$ carboplatin $100 \mu \mathrm{M}$, or combination WNT974 and carboplatin totaling $5 \mu \mathrm{l}$. The plates were incubated at $37^{\circ} \mathrm{C}$ in a humidified $5 \% \mathrm{CO}_{2}$ atmosphere and treated with WNT974 on day 2 and carboplatin on day 6 totaling $48 \mathrm{~h}$ of carboplatin treatment. All patient samples underwent testing with 16 replicates, duplicate assays with 8 replicates each. On day 8 , the cells were lysed and viability was analyzed using the ATPlite luminescence-based assay (PerkinElmer, Waltham, MA, USA) as previously described. ${ }^{9}$ The ascites samples that showed a decrease in ATP concentration after treatment demonstrated no difference from the untreated cells in percent viability with trypan blue staining. Flow cytometry was performed on treated and untreated ovarian cancer ascites cells. Samples showing $\geq 30 \%, 10-30 \%$, and $<10 \%$ reduction in ATP counts compared with the untreated control were deemed WNT974 sensitive, intermediate sensitive, and resistant, respectively.

\section{$\mathrm{IC}_{50}$ and Dose-Response Analysis}

Ascites cells from the WNT974 sensitive patient samples were prepared as above in 96-well ultra-low attachment plates then treated with WNT974 at varying concentrations $(0.25,0.5$, $0.75,1,1.5,1.75,2,2.25,2.5,3$, and $3.5 \mu \mathrm{M})$. After 7 days of treatment with WNT974, viability was analyzed using the same ATPlite luminescence-based assay.

\section{Immunohistochemistry}

To compare treated and untreated patient samples, ascites cells were plated in 6-well ultra-low attachment plates (Corning Costar) at 150000 cells/well then treated with $1 \mu \mathrm{M}$ WNT974 for $48 \mathrm{~h}$. Untreated and treated ascites cells were prepared for IHC staining as previously described. ${ }^{9}$ Formalin-fixed, paraffin-embedded slides from the untreated tumor and an omental metastatic site collected at the time of the initial surgery were used for IHC staining of the tumor samples. Papillary serous histology was confirmed by hematoxylin and eosin staining. Additional slides from the paraffin block were sectioned at $4 \mu \mathrm{m}$ for IHC. Slides were melted in a $60^{\circ} \mathrm{C}$ oven for $1 \mathrm{~h}$ and the sections were cleared in a graded xylene/ethanol series then rinsed in $\mathrm{dH}_{2} \mathrm{O}$. The slides underwent antigen retrieval by placing in pre-heated $0.01 \mathrm{M}$ citric acid pH 6 (LRP6) or $10 \mathrm{mM}$ Tris/1 mM EDTA pH 9 (other antibodies) and by pressure cooking for $10 \mathrm{~min}$ at 15 psi. Pressure was allowed to equilibrate and slides were cooled for $10 \mathrm{~min}$. Sections were treated with 3\% hydrogen peroxide $/ \mathrm{H}_{2} \mathrm{O}$ for $5 \mathrm{~min}$ to suppress endogenous peroxidase and blocked with $3 \%$ goat serum to reduce non-specific binding. Primary antibodies were diluted in Antibody Diluent Buffer (1\% BSA, $1 \mathrm{mM}$ EDTA in PBS) as follows: polyclonal rabbit anti-phosphorylated LRP6 (Bioss Antibodies, Woburn, MA, USA), 1:50, polyclonal rabbit anti-PORCN (Novus Biologicals, Littleton CO, USA), 1:50, monoclonal rabbit anti-E-cadherin (ThermoFisher, Waltham, MA, USA), 1:100, monoclonal rabbit anti-survivin, clone EPR2675 (Abcam, Cambridge, MA, USA), 1:100, monoclonal mouse anti$\beta$-catenin, clone E-5 (Santa Cruz Biotechnology, Dallas, 
TX, USA), 1:750. Primary antibodies were incubated for one hour at room temperature. Control multi-tissue slides were used as a positive control for each staining run. The secondary detection system was EnVision+System HRP-Labeled Polymer Anti-Rabbit or Anti-Mouse (Dako, Carpinteria, CA, USA). DAB substrate (Scytek Laboratories, Logan, UT, USA) was used to visualize antibody-antigen interaction and then counterstained with Mayer's Hematoxylin Solution, dehydrated through alcohols and xylenes, and mounted using permount. Once IHC staining was completed, the intensity of the staining for $\beta$-catenin, E-cadherin, survivin, PORCN, and phosphorylated LRP6 was graded on a scale of 0 (no staining) to 4 (intense staining). The weighted average of the intensity score was calculated by multiplying the percentage of cells at each intensity by the decimal equivalent of that percentage. This weighted average is considered the immunostaining score. ${ }^{20}$ The intensity of each ascites and tumor sample was reported by a pathologist who was blinded to treated $v s$ untreated ascites cells.

\section{Taqman Assays}

For tumor tissue homogenization, samples were weighed and placed in $15 \mathrm{ml}$ conical tubes containing $1.4 \mathrm{~mm}$ ceramic beads (VWR cat. \# 10032-374). The tissue samples were suspended in $35 \mu \mathrm{l}$ of buffer (Qiagen, Valencia, CA, USA) plus $1 \%$ BME per gram of tissue. The samples were then agitated on a MP Biomedicals FastPrep machine for three cycles of $45 \mathrm{~s}$ at full speed. Homogenized samples were stored at $-80^{\circ} \mathrm{C}$ until use. Ascites cell samples were thawed and an aliquot was counted. Cell counts ranged from 30000 to $96 \times 10^{6}$ per sample. Cells were then centrifuged at $200 \mathrm{~g}$ for 2 mins, and the supernatant removed. A minimum of $700 \mu \mathrm{l}$ of buffer plus $1 \%$ BME was added to each sample. When necessary, additional buffer was added to dilute the sample to $\sim 8 \times 10^{6}$ cells $/ \mathrm{ml}$. Cell lysates were stored at $-80^{\circ} \mathrm{C}$ until use.

RNA was extracted from $350 \mu \mathrm{l}$ of tumor or ascites cell lysate using the Norgen Total RNA Purification Kit (Norgen Biotek, Ontario, Canada). Tumor samples were treated with Proteinase K prior to extraction, and the on-column DNase digest was performed according to the manufacturer's instructions. After elution, total RNA was quantified using a Qubit 2.0 fluorometer and RNA HS Assay Kit (Invitrogen, Carlsbad, CA, USA). Reverse transcription was performed on $500 \mathrm{ng}$ of total RNA or $10 \mu \mathrm{l}$ of extracted total RNA in the case of low-yield samples using the High Capacity cDNA Reverse Transcription Kit from Applied Biosystems (Green Island, NY, USA). The reaction was performed in a Bio-Rad T100 thermal cycler programmed according to kit instructions. DNA was extracted from $350 \mu \mathrm{l}$ of tumor or ascites cell lysate using the Qiagen DNeasy Blood \& Tissue Kit. RNase digestion was omitted, but extraction was otherwise performed according to manufacturer's instructions. After elution, DNA was quantified using a Qubit 2.0 fluorometer and dsDNA HS Assay Kit (Invitrogen).
Pre-mixed TaqMan assays were ordered from Applied Biosystems for control genes (ACTB, GAPDH, and FCGRT), PORCN, RSPO2, RSPO3, AXIN2, and Wntless (WLS). Forward and reverse primers and TaqMan probes were designed for the three gene fusions E1F3E (e1)-RSPO2(e2), PTPRK(e1)-RSPO3(e2), and PTPRK(e7)-RSPO3(e2). Positive controls were included via genomic DNA, synthetic oligos (IDT), or cDNA clone (Transomic, Huntsville, AL, USA). For low-yield samples, a 1:5 dilution of cDNA was used. Otherwise, a 1:10 dilution was analyzed. QPCR for all 11 genes: 3 gene fusions, 3 control genes, and RSPO2, RSPO3, AXIN2, WLS, and PORCN expression were performed in triplicate on each cDNA sample. Each $10 \mu \mathrm{l}$ reaction was prepared using TaqMan Universal Master Mix (Applied Biosystems). Samples were run in a 384-well plate on an ABI Prism 7900HT Sequence Detection System running SDS 2.4 software. Control values were computed automatically. Relative quantification of transcripts were calculated first by normalizing to housekeeping genes and then by normalizing for amplicon-specific amplification based on standard curves.

\section{Statistical Analysis}

The ratio of ATP levels (counts per second) for treated $v s$ untreated control ascites cells (percent control) was calculated. Statistical analysis comparing ATP levels was performed using SAS version 9.2. A mean percent viability was calculated from all replicates of the control group receiving no treatment. This value was then divided by itself to give a null value of 1 . The proportion of viability for each treatment group was then compared with the null value of 1 using a one-tailed $t$-test to show the difference in viability. Significance was determined when the $P$-value was $<0.05$. The WNT974 IC $_{50}$ (half maximum inhibitory concentration) was defined as the $\log _{10}$ concentration generating a $50 \%$ reduction in ATP levels when compared with the untreated control. All ATPlite analyses represent 16 replicates and $\mathrm{IC}_{50}$ data represent eight replicates. Mean \pm s.e. is represented in the error bars as detailed in the figure legends. Fisher's exact, $X^{2}$, and student's $t$-test were used to determine the statistical significance of descriptive statistics. A comparison of RSPO2, RSPO3, and PORCN gene expression to ascites sample sensitivity was performed using a two-way ANOVA test. Paired $t$-test was used to calculate changes in AXIN2 levels following treatment with WNT974 compared with matched untreated samples. Significance was assigned when the $P$-value was $<0.05$.

\section{RESULTS}

Ascites samples from 60 patients were analyzed. Patient demographics are outlined in Table 1 . The mean age was 64 years old, and the majority of patients were white (85\%). Fifty $(83.3 \%)$ patients were diagnosed with ovarian cancer and $10(16.7 \%)$ with primary peritoneal cancer. All but one patient had primary disease. RECIST 1.1 was used to determine response rates after chemotherapy. Thirty-two (53.3\%) 
Table 1 Patient demographics

$N=60(\%)$

\section{Race}

White

African American

Hispanic

Mean age $( \pm$ s.d.)

Mean BMI ( \pm s.d.)

Site of malignancy

$$
\text { Ovary }
$$

Primary peritoneal

Histology

Papillary serous

$60(100)$

\section{Disease status \\ Primary \\ Recurrent}

Clinical response to treatment

Complete response

Partial response

13 (21.7)

Progression

Unknown

11 (18.3)

Recurred after complete response

$10(31.3)$

Time to recurrence

Median (range)

$9.5(2-15)$

Clinical status at last follow-up
No evidence of disease
Alive with disease
Deceased
Unknown

$27(45)$

$11(18.3)$

$12(20)$

$10(16.7)$

patients had a complete response and $13(21.7 \%)$ patients had a partial response. Response rates were unable to be determined in $11(18.3 \%)$ patients due to incomplete medical records, death before completing chemotherapy, or the patient is still receiving adjuvant chemotherapy. In those 32 women who experienced a complete response, 10 (31.3\%) recurred with a median time to recurrence of 9.5 months.

Cell viability analysis was performed on all 60 patient ascites samples. Seven patient samples were excluded because there were not enough tumor cells in the thawed samples to

proceed with testing. Figure 1 shows the range, $0-97 \%$, of the decrease in ATP concentration expressed as a percent of untreated control for 53 samples when treated with $1 \mu \mathrm{M}$ WNT974. The results of the ATPlite analysis are grouped into three different response groups in Figure 2. Few samples responded to WNT974 alone, but a large fraction of patient samples showed significant loss of ATP when WNT974 was combined with carboplatin. This ATP loss was greater than treatment with carboplatin alone. Table 2 shows the relationship between the response groups in vitro to their actual clinical response. Despite the limited response to single agent WNT974, 32/51 (63\%) of patient samples showed $\geq 30 \%$ reduction in ATP concentration with combination treatment. Patients with both platinum-sensitive and platinum-resistant disease showed strong response to combination treatment with $73 \%$ and $50 \%$, respectively, showing high levels of ATP reduction. There was no difference in the sensitivity to WNT974 alone between the platinum-sensitive and platinum-resistant patient samples. Only 2 of the 14 (14\%) samples of cells isolated from platinum-resistant patient samples treated with carboplatin alone had a $\geq 30 \%$ reduction in ATP concentration, whereas 12 of the $26(46 \%)$ of the cells isolated from platinum-sensitive patient samples had a $\geq 30 \%$ reduction in ATP concentration, suggesting that there is a correlation between clinical outcomes and in vitro results, although they do not correspond perfectly.

$\mathrm{IC}_{50}$ and dose-response analysis was performed on sensitive patient ascites samples (patient samples that had a $\geq 30 \%$ reduction in ATP concentration). Representative samples are shown in Figure 3 for patients OV-7 and OV-41 treated with a range of doses. The $\mathrm{IC}_{50}$ for OV-7 was 1.14 and $1.76 \mu \mathrm{M}$ for OV-41. When correlating these samples to the results in Figure 1, where $1 \mu \mathrm{M}$ WNT974 was used in the ATPlite analysis, OV-7 shows a 50\% reduction in ATP counts with $1 \mu \mathrm{M}$ WNT974 while there was only a $33 \%$ reduction in ATP count for OV-41 when using $1 \mu \mathrm{M}$ WNT974. A 50\% reduction could be obtained after increasing the dose of WNT974 to $1.75 \mu \mathrm{M}$ as shown in Figure 3.

The ascites samples that showed a decrease in ATP concentration after treatment demonstrated no difference from the untreated cells in percent viability with trypan blue staining, suggesting the WNT974 was not cytotoxic. Flow cytometry demonstrated fewer cells in the G2 phase and more in the G1 and S phases after 2 or 7 days of treatment with WNT974, supporting cell cycle arrest as the mechanism of action of WNT974 (Figure 4).

IHC analysis of $\beta$-catenin, E-cadherin, PORCN, survivin, and phosphorlyated-LRP6 was performed on the primary tumor, a metastatic tumor implant, and both untreated ascites cells and those cells treated with WNT974 only from six different patient samples. $\beta$-catenin membrane expression is shown in Figures $5 \mathrm{a}$. E-cadherin membrane staining shows no difference between the primary tumor and the metastatic site or between the untreated and the treated ascites (Figure 5b). There were similar levels of cytoplasmic PORCN 


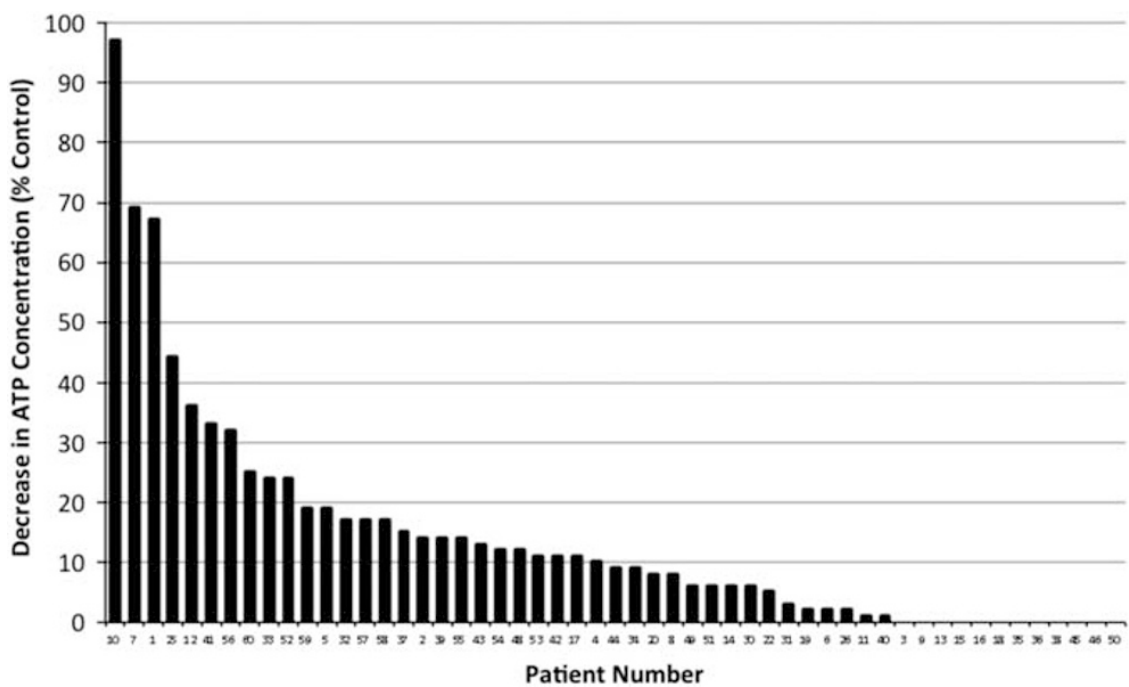

Figure 1 Patient sample sensitivity. Fifty-three ovarian cancer ascites samples were treated with $1 \mu \mathrm{M}$ WNT974 for 7 days. Sensitivity was analyzed using the ATPlite luminescence-based assay.

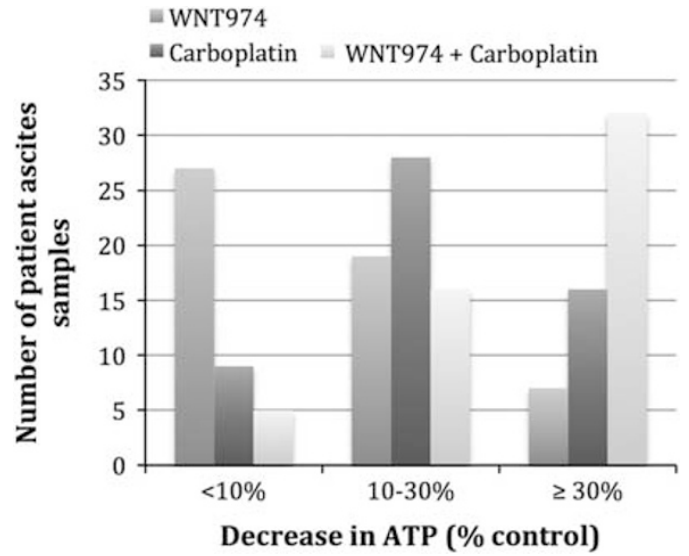

Figure 2 Sensitivity of patient ascites samples to WNT974. Fifty-three ovarian cancer ascites samples were treated with $1 \mu \mathrm{M}$ WNT974, $100 \mu \mathrm{M}$ carboplatin, or a combination of WNT974 and carboplatin. Viability was analyzed using the ATPlite luminescence-based assay. The number of tested samples showing each range of decrease in ATP is shown.

expression in both tumor and ascites obtained from the same patient (Figure 5c). This was also true for both cytoplasmic and nuclear staining of Survivin, although the nuclear staining was stronger than the cytoplasmic staining (Figures $6 a$ and $b$ ). There was a decrease in p-LRP6 in the treated ascites samples compared with the untreated samples, but this was not consistent throughout all six samples (Figures $6 \mathrm{c}$ and $\mathrm{d}$ ). Treatment with WNT974 did not change the expression of $\beta$ catenin, E-cadherin, PORCN, survivin, or phosphorlyatedLRP6 measured by IHC. We did, however, observe that in 20 ascites samples WNT974 treatment decreased the levels of AXIN2 transcript compared with matched untreated ascites samples, confirming that WNT974 does reduce signaling through the Wnt $/ \beta$-catenin pathway $(P=0.0002$; Figure 7$)$.
Table 2 Patient ascites sensitivity after drug treatment compared with the clinically observed response

\begin{tabular}{|c|c|c|c|c|}
\hline \multirow[t]{2}{*}{$\begin{array}{l}\text { Decrease in ATP } \\
\text { (\% Control) }\end{array}$} & $\begin{array}{l}\text { Platinum } \\
\text { sensitive }\end{array}$ & $\begin{array}{l}\text { Platinum } \\
\text { resistant }\end{array}$ & Unknown & \\
\hline & $N=26$ & $N=14$ & $N=13$ & \\
\hline WNT974 & & & & $P=0.6723$ \\
\hline$\geq 30$ & 2 & 3 & 2 & \\
\hline $10-30$ & 10 & 4 & 6 & \\
\hline$<10$ & 14 & 7 & 5 & \\
\hline Carboplatin & & & & $P=0.1557$ \\
\hline$\geq 30$ & 12 & 2 & 2 & \\
\hline $10-30$ & 10 & 10 & 8 & \\
\hline$<10$ & 4 & 2 & 3 & \\
\hline WNT974 & & & & $P=0.3446$ \\
\hline \multicolumn{5}{|l|}{ +carboplatin } \\
\hline$\geq 30$ & 19 & 7 & 6 & \\
\hline $10-30$ & 6 & 5 & 5 & \\
\hline$<10$ & 1 & 2 & 2 & \\
\hline
\end{tabular}

Ascites cells from ovarian cancer patients treated with $1 \mu \mathrm{M}$ WNT974 for 7 days, $100 \mu \mathrm{M}$ carboplatin for $48 \mathrm{~h}$, or combination WNT974 and carboplatin are stratified by their clinical response to platinum-based chemotherapy. Patients were categorized as platinum sensitive or resistant. More patient samples demonstrated a $\geq 30 \%$ reduction in ATP concentration and fewer samples showed a $<10 \%$ reduction in ATP concentration with combination therapy compared with treatment with either single agent alone.

We next asked whether the variability in WNT974 sensitivity could be explained by changes in gene expression in the ascites samples. We measured expression of WNT974's target, PORCN and key Wnt pathway genes RSPO2 and 

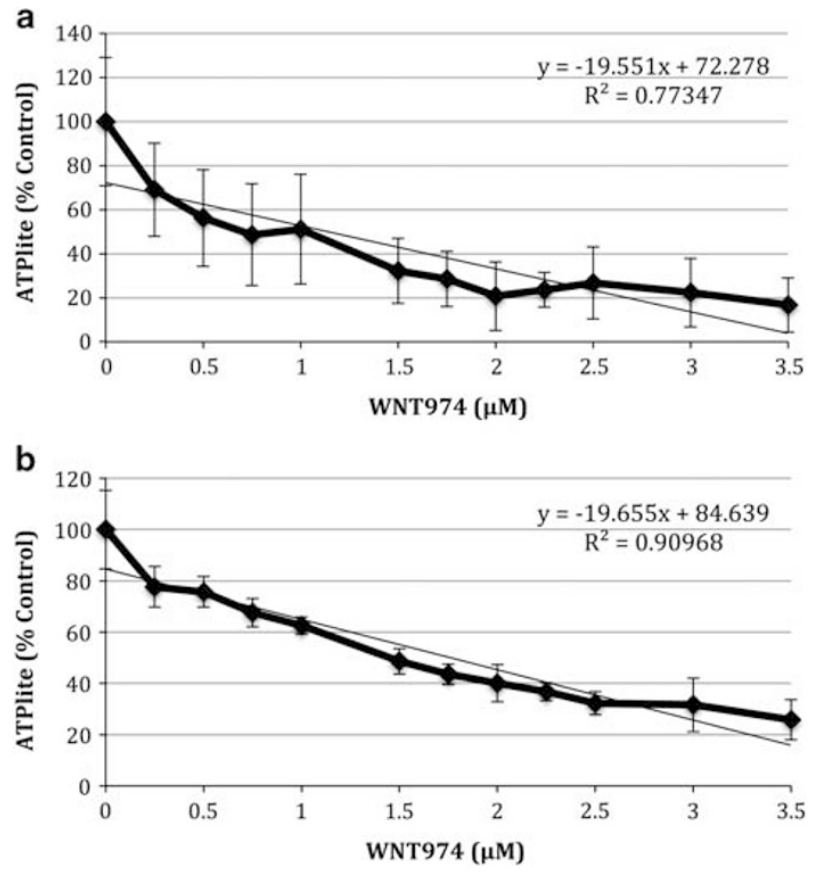

Figure 3 WNT974 IC 50 dose-response curves. Tumor cells isolated from the ascites of patients OV-7 (a) and OV-41 (b) were treated with WNT974 $(0.25$ to $3.5 \mu \mathrm{M})$ for 7 days. Sensitivity was measured with ATPlite. The $I_{50}$ value was determined. Error bars represent s.e.m. for eight replicates.

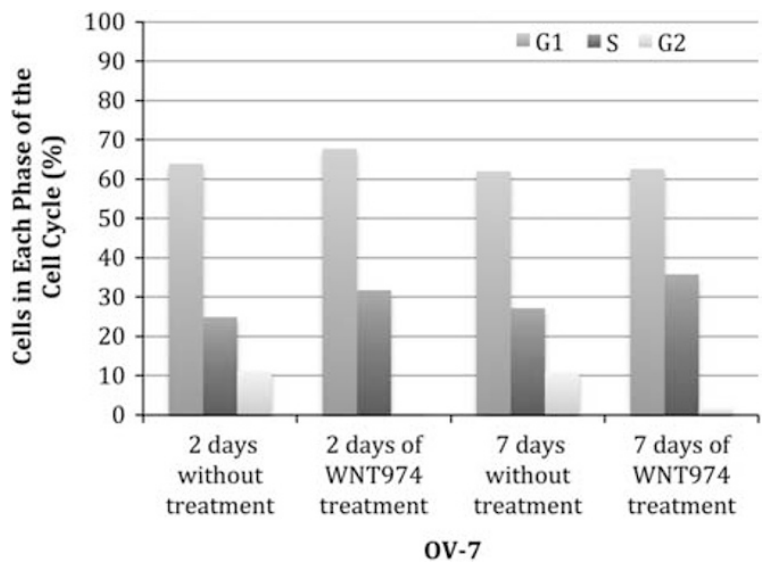

Figure 4 Cell cycle analysis of patient ascites samples. Flow cytometry was performed on patient ascites samples treated with $1 \mu \mathrm{M}$ WNT974 and those left untreated after 2 days and 7 days. A representation of the results is shown using ascites cells from patient OV-7. Treatment caused a decrease in the number of cells in the G2 phase and an increase in the number of cells in the $\mathrm{G} 1$ and $\mathrm{S}$ phases after both 2 and 7 days of treatment.

RSPO3, and three previously characterized fusions involving RSPO2 and RSPO3, which are thought to increase levels of those transcripts. Using custom Taqman assays, we measured expression of these genes and fusions, and assessed whether expression correlated with sensitivity to WNT974 (Figure 8). Analysis was done on 40 matched ascites and tumor samples
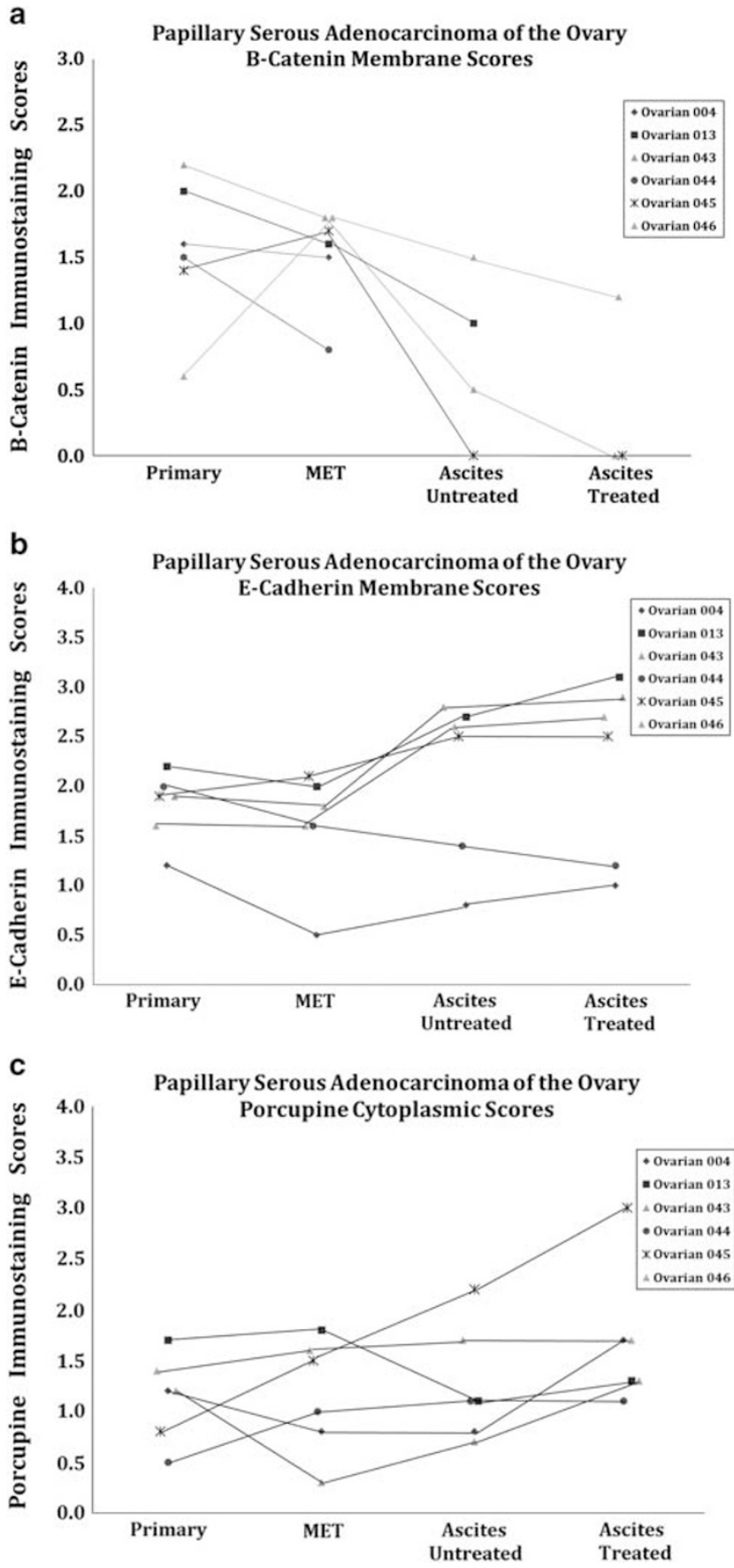

Figure 5 Immunohistochemical analysis of $\beta$-catenin, E-cadherin, and PORCN. IHC analysis of $\beta$-catenin (a), E-cadherin (b), and PORCN (c) was performed on the primary tumor, a metastatic tumor implant (MET), and both untreated and WNT974-only treated ascites tumor cells from six different patient samples. The intensity of staining with $\beta$-catenin and E-cadherin ( $\mathbf{a}$ and $\mathbf{b}$ ) showed similar levels of membranous protein expression among both tumor and ascites cells obtained from the same patients. There were similar levels of PORCN cytoplasmic protein (c) expression among both tumor and ascites cells obtained from the same patient. IHC, immunohistochemistry. 

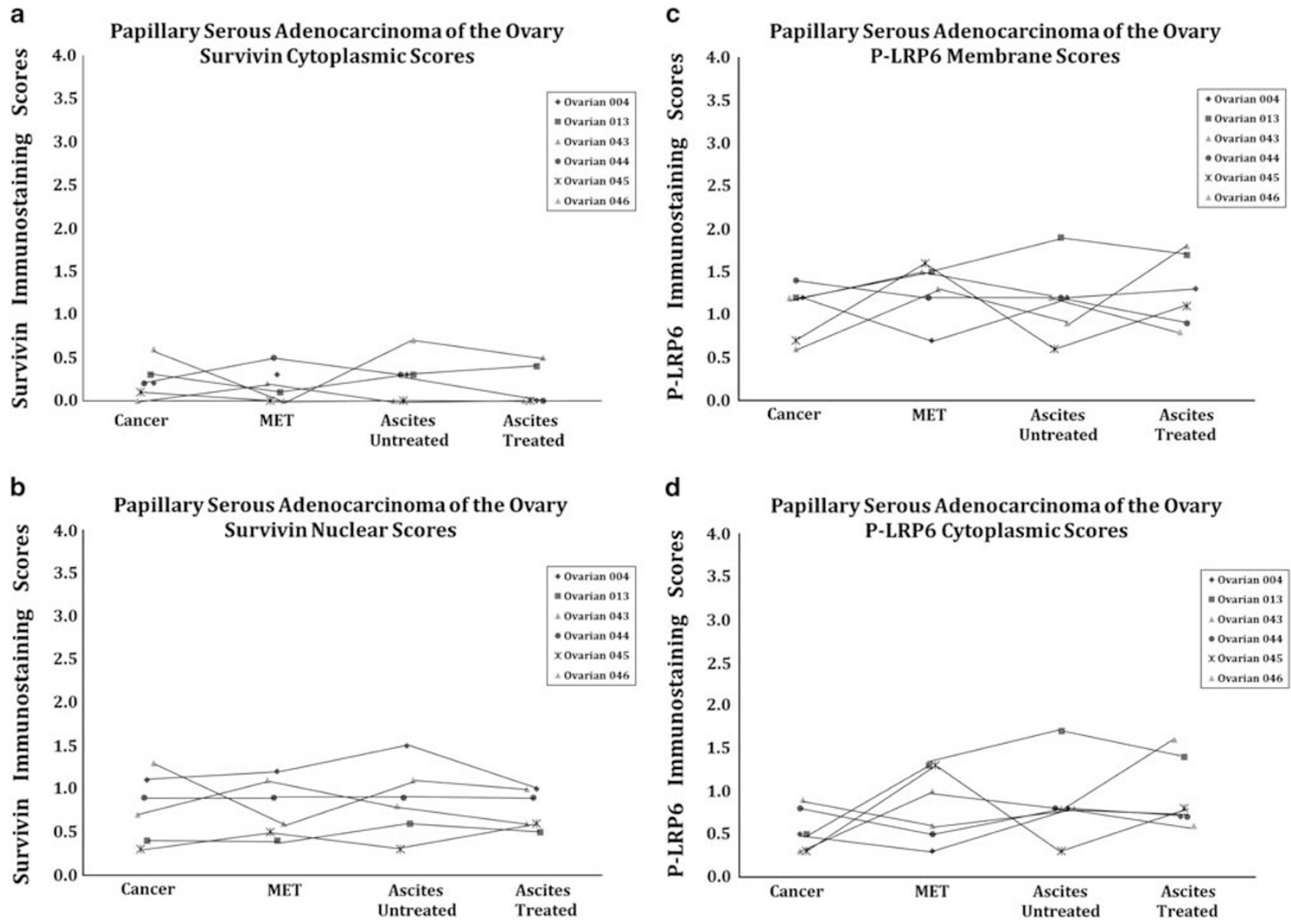

Figure 6 Immunohistochemical analysis of survivin and p-LRP6. IHC analysis of survivin and p-LRP6 was performed on the primary tumor, a metastatic tumor implant (MET), and both untreated and WNT974-only treated ascites tumor cells from six different patient samples. There was similar levels of cytoplasmic (a) and nuclear (b) survivin protein expression among both tumor and ascites cells obtained from the same patients. There were similar levels of p-LRP6 membranous (c) and cytoplasmic (d) protein expression among both tumor and ascites cells obtained from the same patients. IHC, immunohistochemistry.

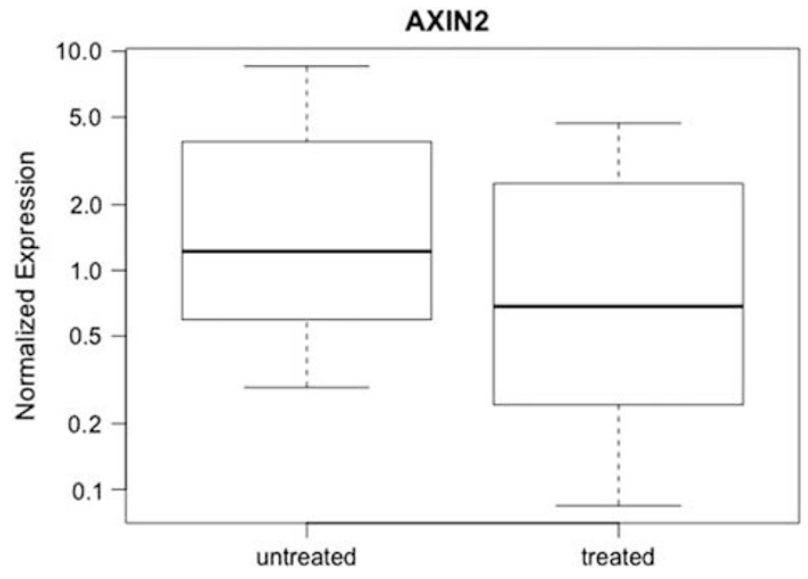

Figure 7 AXIN2 transcript levels in response to WNT974 treatment. The level of AXIN2 transcript was measured in 20 samples of both untreated and matched ascites treated with WNT974. There was a decreased level of AXIN2 transcript in treated ascites confirming that WNT974 reduces signaling through the $\mathrm{Wnt} / \beta$-catenin pathway. Paired $t$-test was used to calculate changes in AXIN2 levels following treatment with WNT974 compared with matched untreated samples. and an additional 13 ascites samples without matching tumor tissue. We found that RSPO fusions were detectable in only one sample, indicating that they do not play a role in these patient samples. In both ascites and tumor cells, an increase in RSPO3 expression was associated with sensitivity to the combination of WNT974 and carboplatin, but not carboplatin or WNT974 alone (Figure 8a). Similarly, increased WLS expression in ascites correlated with sensitivity to WNT974 alone $(P=0.0399)$, trended with sensitivity to carboplatin alone and to the combination treatment $(P=0.065)$, but WLS expression in tumors did not correlate with sensitivity (Figure 9). This suggests an upregulation of the Wnt/ $\beta$-catenin pathway in platinum-resistant ovarian cancer, particularly in metastatic cells. This supports previous studies showing upregulation of the $\mathrm{Wnt} / \beta$-catenin pathway in platinum-resistant ovarian cancer. ${ }^{9,14}$ In addition, PORCN gene expression correlates with ascites cell sensitivity to WNT974 (Figure 8b). Higher PORCN expression was measured in the untreated ascites samples that produced a $\geq 30 \%$ reduction in ATP when treated with WNT974 

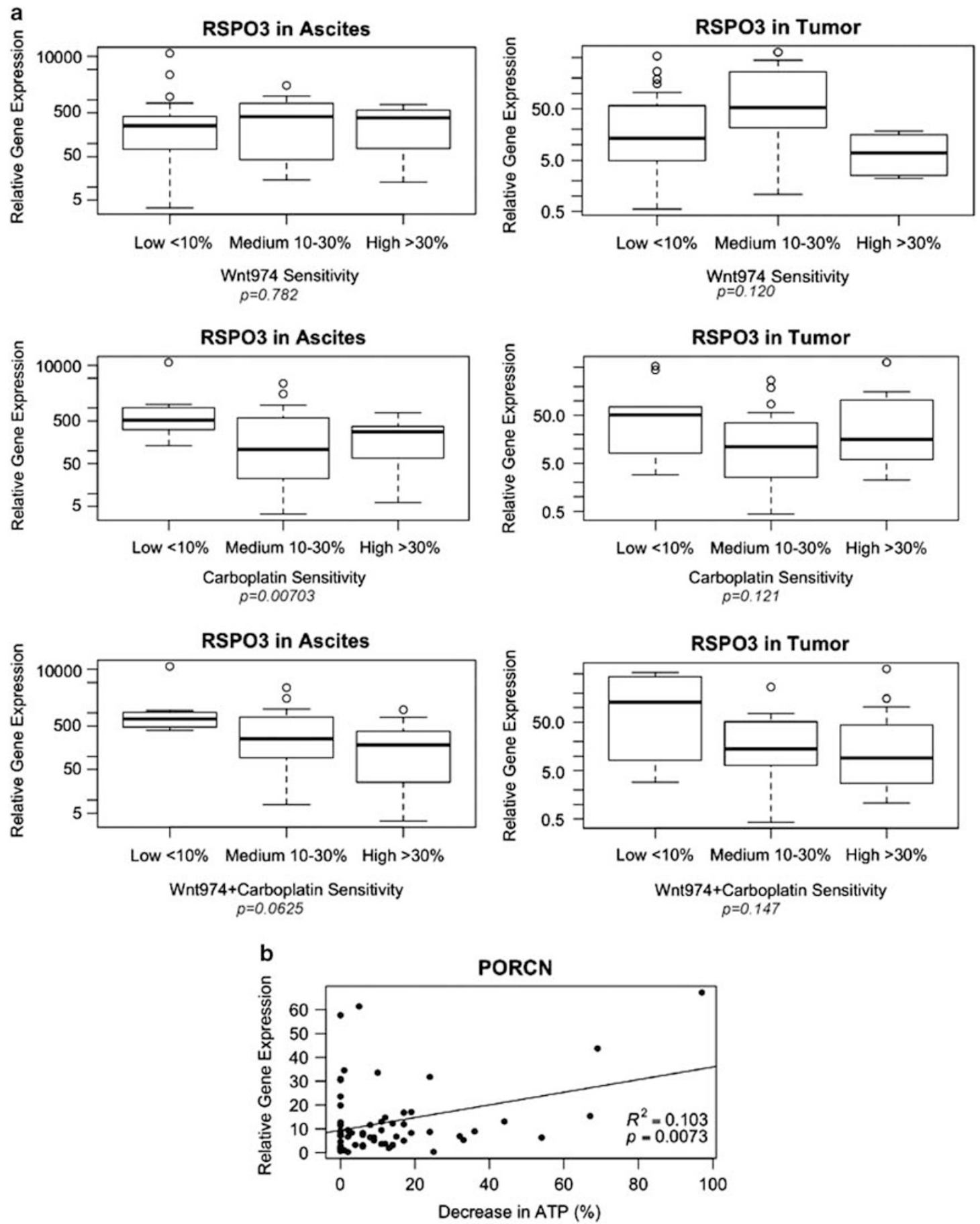

Figure 8 Gene expression in ovarian cancer patient samples. (a) RSPO3 expression before treatment with carboplatin and WNT974 or carboplatin only is compared with the response in ascites cells after treatment. High sensitivity indicates a $\geq 30 \%$ reduction in ATP concentration, medium sensitivity indicates a $10-30 \%$ reduction, and low sensitivity indicates a $<10 \%$ reduction. Samples with the lowest response to WNT974 and carboplatin had higher RSPO3 expression at baseline. (b) PORCN gene expression in untreated ascites cells is compared with ascites sensitivity to WNT974. Higher PORCN expression was correlated with greater decrease in ATP concentration. A Pearson's correlation between PORCN and viability after WNT974 treatment was calculated, and the $P$-value calculated using default $\mathrm{R}$ parameters.

compared with those ascites samples that did not show a drop in ATP after WNT974 treatment $(P=0.0073)$. This suggests that cells with higher endogenous levels of PORCN and WLS are more sensitive to WNT974.

\section{DISCUSSION}

The Wnt $/ \beta$-catenin pathway has been shown to contribute to ovarian cancer initiation, metastasis, chemoresistance, and recurrence. ${ }^{9,11,12}$ Evidence supports the presence of cancer 

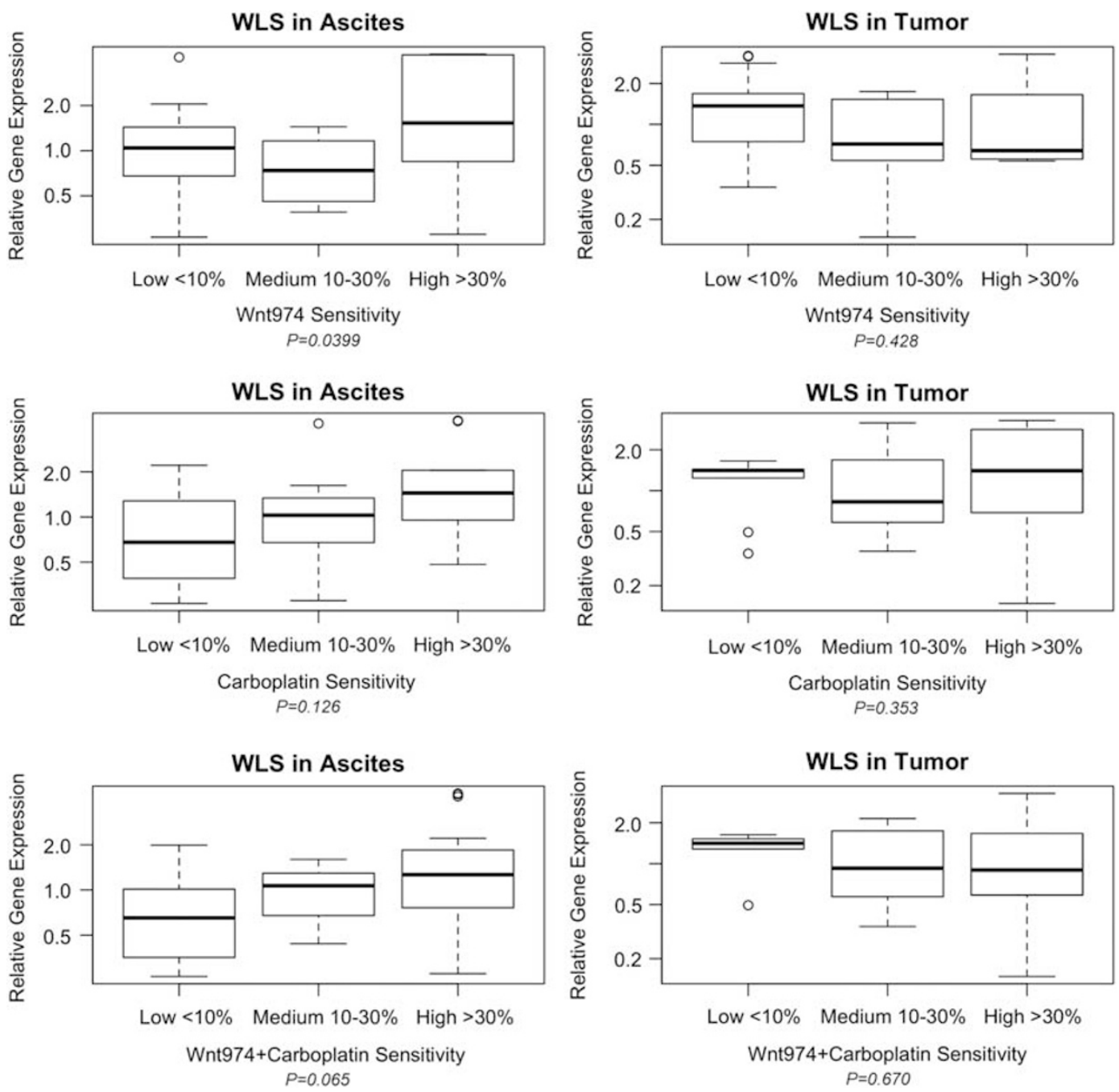

Figure 9 WLS expression in ovarian cancer ascites and tumor. Increased WLS expression was seen in ascites samples sensitive to WNT974 alone. WLS expression only trends with sensitivity to carboplatin alone and to combination WNT974+carboplatin treatment. In tumor samples, WLS expression did not correlate with sensitivity.

stem cells (CSCs) in the development of tumor chemoresistance and recurrence, and upregulation of the $\mathrm{Wnt} / \beta$-catenin pathway found in CSCs is thought to contribute to CSC maintenance. ${ }^{9,21}$ Increased activity of the $\mathrm{Wnt} / \beta$-catenin pathway can result from upregulation at different steps in the signaling pathway: overexpression of the cell surface receptors LRP5, LRP6, and Frizzled or increased activity of the Wnt target genes survivin, cyclin D1, Axin2, and c-myc. ${ }^{9,13,22}$ A crucial component necessary for Wnt ligand transport, secretion, and activity is PORCN, which has been identified as a potential target to inhibit $\mathrm{Wnt} / \beta$-catenin signaling. ${ }^{15}$

Wnt pathway inhibitor success has been limited because of a narrow therapeutic window for activity since normal tissue stability requires $\mathrm{Wnt} / \beta$-catenin signaling and a lack of predictive biomarkers of response. WNT974 inhibits Wnt $/ \beta$ catenin signaling through inhibition of the PORCN enzyme, which causes a decrease in secretion of Wnt ligands. The prevention of Wnt ligand secretion after treatment with WNT974 results in decreased phosphorylation of the surface receptor LRP6 and decreased expression of Wnt target genes like AXIN2. ${ }^{16,17}$ WNT974 has shown anti-tumor activity in pancreatic adenocarcinoma and head and neck squamous cell cancer. Jiang et all ${ }^{17}$ identified both WNT974 sensitive tumor cells and a potential predictive biomarker after demonstrating tumor growth inhibition through cell cycle arrest in in vivo pancreatic tumors treated with WNT974 with inactivating mutations in the RNF43 gene. Wnt/ $\beta$-catenin signaling inhibition was shown through decreased expression of the target genes Axin2 and c-myc. Liu et al ${ }^{16}$ found a dosedependent tumor growth delay in human head and neck squamous cell cancer xenografts treated with WNT974. Evidence of $\mathrm{Wnt} / \beta$-catenin signaling inhibition was shown with decreased Axin2 expression and prevention of LRP6 
receptor phosphorylation. They also discovered a correlation between a loss of function Notch1 gene mutation and WNT974 sensitivity, which may also indicate a potential predictive biomarker for WNT974.

The presence of ascites is a predictor of poor outcomes in women diagnosed with ovarian cancer. ${ }^{10}$ Certain populations of tumor cells in ascites show CSC-like properties, chemoresistance, and the ability to promote distant metastasis and recurrence. ${ }^{10}$ The ascites fluid creates a microenvironment containing the necessary components to promote tumor cell growth. ${ }^{10}$ Since ascites are often present at the time of diagnosis and recurrence, this fluid provides an ideal opportunity to evaluate these tumor cells and advance our understanding of ovarian cancer pathogenesis.

We evaluated the effect of WNT974 on primary ovarian cancer ascites cells isolated from 53 patient samples with papillary serous ovarian cancer. Trypan blue staining showed no change in the percent viability after treatment with WNT974, suggesting that it did not cause cytotoxicity to the cells. Flow cytometry results support cell cycle arrest as the mechanism of action for WNT974 in ovarian cancer ascites cells. IHC analysis of E-cadherin showed similar levels of expression in both the untreated and treated ascites cells, suggesting a lack of epithelial-to-mesenchymal transition. Reduction in AXIN2 expression indicates inhibition of the $\mathrm{Wnt} / \beta$-catenin pathway. Cell cycle arrest was demonstrated on ATPlite analysis with a $\geq 30 \%$ reduction in ATP concentration in $13 \%$ of patient samples treated with WNT974 only and 30\% of patient samples treated with carboplatin only. The number of samples that showed a $\geq 30 \%$ reduction in ATP concentration increased to $60 \%$ when WNT974 was combined with carboplatin treatment. Similar IHC staining of the Wnt pathway proteins $\beta$-catenin, survivin, and phosphorlyated-LRP6 and the PORCN enzyme between tumor and both untreated and treated ascites cells suggests cytostasis rather than cytotoxicity with WNT974 treatment.

Despite a previous preliminary report of RSPO fusion transcripts in ovarian cancer, we found little evidence for RSPO fusions in either tumor or ascites samples. ${ }^{19}$ Our efforts to reveal gene expression patterns that correlate with sensitivity to Wnt974 showed that there was also no correlation between RSPO3 gene expression and sensitivity to WNT974 alone but increased RSPO3 levels were associated with response to the combination of WNT974 and carboplatin, suggesting that WNT974 makes chemotherapy-resistant cells more sensitive to carboplatin. We did demonstrate increased $\mathrm{RSPO} 3$ gene expression in samples resistant to carboplatin in support of previous studies demonstrating upregulation of the $\mathrm{Wnt} / \beta$-catenin pathway in platinumresistant cancer cells. ${ }^{9,14}$ PORCN expression may be a potential indicator for patients who would respond to WNT974 because higher PORCN expression was seen in the patient ascites samples with $\geq 30 \%$ reduction in ATP on ATPlite analysis.
The WLS gene is a key modulator of Wnt protein expression that is overexpressed in some ovarian cancers. ${ }^{23}$ We observed that WLS expression was lower in ascites compared with tumor but that increased WLS expression in ascites correlated with sensitivity to the WNT974+carboplatin combination.

There are several strengths of this study including the large number of samples tested, specifically the 40 matched tumor and ascites specimens. All but one of the samples collected in this study were obtained at the time of primary diagnosis. Comparing both ascites and tumor gene expression allowed us to determine that biomarkers of drug sensitivity were similar in ascites and tumor. Using ascites cells in this study allowed us to study their microenvironment which may create chemoresistance. ${ }^{24}$ In the future, we plan to evaluate samples from patients with recurrent disease since $\mathrm{Wnt} / \beta$-catenin signaling may be further upregulated in patients with recurrent disease. ${ }^{9,14}$

Wnt inhibitors are now being evaluated in several clinical trials. A Phase I trial using LGK974, renamed WNT974 by Novartis, opened in 2011 for patients with pancreatic adenocarcinoma, BRAF mutant colorectal cancer (CRC), and other tumors with documented genetic mutations upstream in the $\mathrm{Wnt} / \beta$-catenin signaling pathway (NCT01351103). Patients with BRAF-mutated metastatic CRC and other Wnt pathway mutations are eligible for a Phase Ib/II trial evaluating the safety and activity of the combination of WNT974, the RAF kinase inhibitor LGX818, and cetuximab (NCT02278133). This clinical trial evaluates multiple targeted therapies, which may provide better clinical outcomes than a single agent. Phase II trials combining these targeted agents with standard chemotherapy may be warranted given our finding of improved response with a combination of WNT974 and carboplatin.

\section{CONCLUSION}

The Wnt/ $\beta$-catenin pathway is an important signaling pathway that lends itself to pharmacologic intervention through mechanisms different than cytotoxic chemotherapy. Through modification of proteins on the cell surface, in the cytoplasm, and in the nucleus, we have shown activity of the Wnt/ $\beta$-catenin pathway inhibitor WNT974 in patients with papillary serous ovarian cancer. Genetic analysis of these ascites samples has identified a potential predictive biomarker for patients that may respond to WNT974 in elevated PORCN expression. The toxicity of WNT974 in patients with peritoneal disease is under investigation. Ultimately, the combination of targeted agents like WNT974 with cytotoxic chemotherapy may prove to be an effective option for patients with recurrent ovarian cancer.

\section{ACKNOWLEDGMENTS}

We would like to thank Ashwini A Katre, Jeffrey C Sellers, Patsy G Oliver,

Debbie Della Manna, and our colleagues at HudsonAlpha: Ryne Ramaker and Brittany Lasseigne for their technical assistance and mentorship. This work was 
supported by a grant from the Norma Livingston Ovarian Cancer Foundation. WNT974 was obtained from Novartis Institutes for Biomedical Research under a material transfer agreement.

\section{DISCLOSURE/CONFLICT OF INTEREST}

The authors declare no conflict of interest.

1. Siegel RL, Miller KD, Jemal A. Cancer statistics, 2015. CA Cancer J Clin 2015;65:5-29.

2. McGuire WP, Hoskins WJ, Brady MF et al. Cyclophosphamide and cisplatin compared with paclitaxel and cisplatin in patients with stage III and stage IV ovarian cancer. N Engl J Med 1996;334:1-6.

3. Sandercock J, Parmar MK, Torri $V$ et al. First-line treatment for advanced ovarian cancer: paclitaxel, platinum and the evidence. Br J Cancer 2002;87:815-824.

4. Ozols RF, Bundy BN, Greer BE et al. Phase III trial of carboplatin and paclitaxel compared with cisplatin and paclitaxel in patients with optimally resected stage III ovarian cancer: a Gynecologic Oncology Group study. J Clin Oncol 2003;21:3194-3200.

5. Armstrong DK, Bundy B, Wenzel L et al. Intraperitoneal cisplatin and paclitaxel in ovarian cancer. N Engl J Med 2006;354:34-43.

6. Katsumata N, Yasuda M, Takahashi F et al. Dose-dense paclitaxel once a week in combination with carboplatin every 3 weeks for advanced ovarian cancer: a phase 3, open-label, randomised controlled trial. Lancet 2009;374:1331-1338.

7. Burger RA, Brady MF, Bookman MA et al. Incorporation of bevacizumab in the primary treatment of ovarian cancer. N Engl J Med 2011;365: 2473-2483.

8. Hoskins WJPC, Young RC, Barakat RR et al. Principles and Practice of Gynecologic Oncology, 4th (edn). Lippincott Williams \& Wilkins: Philadelphia, PA, USA, 2005.

9. Arend RC, Londono-Joshi Al, Samant RS et al. Inhibition of Wnt/betacatenin pathway by niclosamide: a therapeutic target for ovarian cancer. Gynecol Oncol 2014;134:112-120.

10. Ahmed N, Stenvers KL. Getting to know ovarian cancer ascites: opportunities for targeted therapy-based translational research. Front Oncol 2013;3:256.
11. Verras $M$, Sun $Z$. Roles and regulation of Wnt signaling and betacatenin in prostate cancer. Cancer Lett 2006;237:22-32.

12. Wend $P$, Holland JD, Ziebold $U$ et al. Wnt signaling in stem and cancer stem cells. Semin Cell Dev Biol 2010;21:855-863.

13. Arend RC, Londono-Joshi Al, Straughn JM Jr et al. The Wnt/betacatenin pathway in ovarian cancer: a review. Gynecol Oncol 2013;131: 772-779.

14. Su HY, Lai HC, Lin YW et al. Epigenetic silencing of SFRP5 is related to malignant phenotype and chemoresistance of ovarian cancer through Wnt signaling pathway. Int J Cancer 2010;127:555-567.

15. Proffitt KD, Madan B, Ke $Z$ et al. Pharmacological inhibition of the Wnt acyltransferase PORCN prevents growth of WNT-driven mammary cancer. Cancer Res 2013;73:502-507.

16. Liu J, Pan S, Hsieh MH et al. Targeting Wnt-driven cancer through the inhibition of Porcupine by LGK974. Proc Natl Acad Sci USA 2013;110: 20224-20229.

17. Jiang X, Hao HX, Growney JD et al. Inactivating mutations of RNF43 confer Wnt dependency in pancreatic ductal adenocarcinoma. Proc Natl Acad Sci USA 2013;110:12649-12654.

18. Binnerts ME, Kim KA, Bright JM et al. R-Spondin1 regulates Wnt signaling by inhibiting internalization of LRP6. Proc Natl Acad Sci USA 2007;104:14700-14705.

19. Cardona GMBK, Portale J, Gaffney D et al. Abstract 2408: Identification of R-Spondin fusions in various types of human cancer. Cancer Res 2014;74:2408101158/1538-7445AM2014-2408 2014.

20. Poczatek RB, Myers RB, Manne U et al. Ep-Cam levels in prostatic adenocarcinoma and prostatic intraepithelial neoplasia. J Urol 1999;162:1462-1466.

21. Ricci F, Bernasconi S, Perego P et al. Ovarian carcinoma tumorinitiating cells have a mesenchymal phenotype. Cell Cycle 2012;11: 1966-1976.

22. Barbolina MV, Burkhalter RJ, Stack MS. Diverse mechanisms for activation of Wnt signalling in the ovarian tumour microenvironment. Biochem J 2011;437:1-12.

23. Stewart J, James J, McCluggage GW et al. Analysis of wntless (WLS) expression in gastric, ovarian, and breast cancers reveals a strong association with HER2 overexpression. Mod Pathol 2015;28:428-436.

24. Domcke $S$, Sinha R, Levine DA et al.. Evaluating cell lines as tumour models by comparison of genomic profiles. Nat Commun 2013;4:2126. 